\title{
Usage of Cellulose Diacetate as Sorption Material for Fluorescent Analysis of PAH
}

\author{
ANNA V. STRAŠKO ${ }^{1 \star}$, TAMARA I. GUBINA ${ }^{1}$, ANNA B. SHIPOVSKAYA ${ }^{1,2}$, \\ ANDREY G. MEL'NIKOV ${ }^{1}$ and OLGA N. MALINKINA ${ }^{1,2}$ \\ 'Department of Ecology and Service, Saratov State Technical University \\ Named After Yuri Gagarin, 77 Polytechnicheskaya St., Saratov 410054, Russian Federation \\ ${ }^{2}$ Institute of Chemistry, Saratov State University named after N.G. Chernyshevsky, \\ 83 Astrakhanskaya St., Saratov 410012, Russian Federation. \\ ${ }^{*}$ Corresponding author E-mail: strashkoanna@yandex.ru
}

http://dx.doi.org/10.13005/ojc/300312

(Received: August 01, 2014; Accepted: September 03, 2014)

\begin{abstract}
Solid-phase matrices made of cellulose diacetate (CDA) as films and fibers were prepared. The CDA film matrices were found to be characterized by a negative value of the surface potential $(-32 \pm 2 \mathrm{mV})$ while the fibers had positive ones $(+419 \pm 1 \mathrm{mV})$. The possibility of using these matrices as sorption materials for luminescence analysis of polycyclic hydrocarbons in water-ethanol and micellar media is shown. Modification of such polymeric CDA matrices with surfactants of various natures improves their sorptive capacity and hydrocarbon recovery, and increases the sensitivity of luminescent analysis.
\end{abstract}

Key words: Cellulose diacetate, Fluorescent analysis, Polycyclic aromatic hydrocarbons, Ethanolic media, Aqueous micellar solutions, Surfactants.

\section{INTRODUCTION}

Fluorescent techniques are widely used in various applications and studies, for example, in analytical assays of complex-built organic molecules, in particular, polycyclic aromatic hydrocarbons $(\mathrm{PAH})^{1-3}$. Most organic substances have their luminescence spectra as sets of broad diffuse bands which considerably get narrower as temperature is lowered down to $\sim 77 \mathrm{~K}$. Luminophores are identified and quantified by these typical quasi-line spectra.
Many analytes, especially PAH compounds, occur in the environment in very small quantities, and their luminescence analysis requires pre-concentration of the solutions.

Because of the complexity of lowtemperature measurements and the need to raise the concentration of working solutions in analysis of trace amounts of PAH, micellar-stabilized and solid-phase luminescence (SPL) are used. The application of SPL enables one to combine 
solid-phase extraction with getting a signal in the sorbent phase ${ }^{4-10}$. This method allows observing the fluorescence of substances previously adsorbed on solid matrices, just at room temperature. Hitherto, zeolites $^{4}$, quartz glass ${ }^{5}$, foamed polyurethanes ${ }^{6,7}$, nylon membranes ${ }^{8}$, silver nanoparticles ${ }^{9}$ or gold ones $^{10}$, etc. have been conventionally used as matrices for luminophore sorption in the SPL method.

Earlier ${ }^{11}$ we studied the possibility of using cellulose in the form of filter paper as a matrix to detect and analyze PAHs by SPL, this being a solid particulate material of microfiber structure, whose fibers had the ability to immobilize various substances on their surface. PAH sorption on the cellulose matrix increases the quantum yield of the luminophore. To improve the efficiency of PAH concentration, sorption of the reagent was performed from aqueous micellar solutions of surfactants. According to Refs ${ }^{7}, 12$, sorption under these conditions promotes not only solubilization of the luminophore in surfactant micelles but modification of the surface of the sorbent with these substances. It has been found that modification of the cellulose matrix with surfactants of various natures raises the analytical signal of the adsorbed substances, increases the intensity of SPL, and decreases the detection limit of PAH. With pyrene as an example it was shown that, when this PAH was sorbed onto cellulose from the micellar medium of a cationic surfactant, the observed value of the maximum relative fluorescence intensity was higher in comparison with that in the micellar medium of an anionic surfactant.

In view of the high efficiency of the cellulose matrix in analytical assays of PAH by the SPL method, it seems reasonable to use cellulose derivatives for these purposes, in particular, its acetic esters, i.e. cellulose acetates. Polymeric materials made of cellulose acetates are known to have high sorption capacity and to be used as sorbents, filters, and membranes in various industries ${ }^{13-18}$. Depending on the method of formation, cellulose acetate materials can be prepared in various morphological forms such as films and fibers, which allow one to adjust the surface characteristics of such a polymeric sorbent. Besides, the usage of these large-scale artificial polymers in making solid-phase matrices for SPL is relevant and economically feasible due to the annual reproducibility, availability, large reserves, and low cost of the raw material as well as the outlook of their application.

The aim of this work was to study the possibility of using CDA films and fibers as a matrix for luminescence analysis of PAH in ethanolic and water-micellar media, to evaluate the efficiency of modifying these sorption materials with surfactants of various natures.

\section{EXPERIMENTAL}

\section{Preparation of sorption matrices}

CDA matrices of two morphological forms, namely, films and fibers, were used in our study. For their preparation, a commercial sample of bulk CDA with a viscosity-average molecular weight of 77 $\mathrm{kDa}$, an acetylation degree of $55 \%$, and a moisture content of $3 \%$ was taken (Technofilter Ltd., Russian Federation).

Films were cast under standard conditions by placing a 2 wt. \% CDA solution in an acetone : water ( $95: 5$, analytical grade) mixture followed by solvent evaporation. The solution was applied onto mirror flat glasses previously degreased with ethanol and acetone, using a glass round spinneret. The completeness of solvent removal was judged by changes in the weight. The film thickness was $45 \pm 5$ $\mu \mathrm{m}$. According to SEM data, the film structure is a continuous "lacy" polymeric network with pore sizes $\sim 100-500$ nm (Fig. 1 a).

Fibers were fabricated by electrospinning from 11 wt. \% solutions of a mixture of CDA with polyethylene oxide (PEO) (90:10) in 70\% acetic acid (reagent grade). The experimental device for fiber electrospinning included a steel capillary nozzle with an inner diameter of $0.8 \mathrm{~mm}$, a power source, a micropump dispenser, and a receiving (precipitation) electrode as a horizontal ground plane. The surface density of our non-woven electrospun fibers was $18.5 \mathrm{~g} / \mathrm{m}^{2}$. According to SEM data, this matrix has a highly ordered stacking of fibers with diameters within $300-3,000 \mathrm{~nm}$ (Fig. 1 b).

\section{MATERIALS}

Pyrene of the "Purum" brand (Fluka, Merck Schuchardt OHG, Germany, Hohenbrunn) was 
chosen as a PAH. The content of the main substance was $96 \%$. Water-ethanol and water-micellar solutions with a pyrene concentration of $2.24 \times 10^{-5} \mathrm{M}$ were used in our experiments.

The solid CDA matrix was modified with various surfactants, namely, the anionic sodium dodecyl sulfate, SDS (LenReaktiv, Russian Federation, St. Petersburg), the cationic cetyltrimethylammonium bromide, CTAB (Sigma, Alfa Aesar, A. Jonson Matthey Company, Great Britain), and the non-ionic polyoxyethylene (10) mono-4-isooctylphenyl ester, TX-100 (Sigma, New Jersey, Belgium). The content of pure material in all surfactants was $98-99 \%$.

Ethanolic (with $\mathrm{C}_{2} \mathrm{H}_{5} \mathrm{OH}$ concentration of $48 \%$ ) and water-micellar solutions of SDS, CTAB, and TX-100 (a surfactant concentration of $10^{-2} \mathrm{M}$ ) were prepared, which a sample of pyrene was quantitatively dissolved in. The prepared solutions were used as the sorbate. Pyrene sorption was performed in a dynamic mode. $10 \mathrm{ml}$ of a solution was passed five times through a CDA film or fiber and its fluorescence was measured before and after this sorption. For fluorescent $\mathrm{PAH}$ analysis on a polymeric sorbent, the matrix was pre-dried at room temperature.

\section{Methods}

Fluorescent analysis was performed on an LS 55 Perkin-Elmer fluorescent spectrometer (Perkin Elmer Life and Analytical Sciences, Inc., USA) with the Monk-Gillieson monochromators. A xenon lamp operating in pulsed mode with a $50 \mathrm{~Hz}$ frequency served as the source of radiation. Measurements were carried out within a spectral range of $350-450$ $\mathrm{nm}$, with a wavelength setting accuracy $1 \mathrm{~nm}$. The excitation wavelength was $320 \mathrm{~nm}$. The scanning rate was $100 \mathrm{~nm} / \mathrm{min}$. To measure pyrene fluorescence in solution and on the matrix, standard $1-\mathrm{cm}$ quartz cuvettes and solid sample holders were used, respectively.

The surface morphology of solid-phase matrices was evaluated by SEM on a MIRA \I LMU scanning microscope (Tescan, Czech Republic) at $8 \mathrm{kV}$ voltage and $60 \mathrm{pA}$ conductive current. A $5-\mathrm{nm}$ gold layer was sprayed onto all samples with a K450X Carbon Coater (Germany): the spraying current was $20 \mathrm{~mA}$, the spraying duration $1 \mathrm{~min}$. The surface potential was measured on a Surface Potential Sensor (Nima KSV, Finland).

\section{RESULTS AND DISCUSSION}

The intensity of pyrene fluorescence in water-ethanol and water-micellar solutions and on solid-phase matrices based on CDA (films and fibers) was measured.

The intensity of pyrene fluorescence after sorption on the CDA-based matrices from waterethanol solutions of this $\mathrm{PAH}$ reduces, but not very much. This indicates not high sorption capacity of CDA with respect to pyrene in ethanolic solution.

When pyrene was dissolved in an aqueous micellar solution of surfactants the fluorescence intensity of this $\mathrm{PAH}$ increased in comparison with its water-ethanolic solutions. The highest fluorescence signal of pyrene was observed in the solutions of

Table 1: Effect of the sorbate composition on the polarity index of pyrene in the source solutions and in an adsorbed state on solid-phase CDA matrices and on the hydrocarbon recovery degree

\begin{tabular}{|c|c|c|c|c|c|}
\hline \multirow[t]{3}{*}{$\begin{array}{l}\text { Sorbate } \\
\text { composition }\end{array}$} & \multicolumn{3}{|c|}{$\begin{array}{l}\text { Pyrene polarity index } \\
\qquad I_{1} / I_{3} \text { (arb. un.) }\end{array}$} & \multirow{2}{*}{\multicolumn{2}{|c|}{$\begin{array}{c}\text { Pyrene recovery } \\
R(\%)\end{array}$}} \\
\hline & \multirow{2}{*}{$\begin{array}{c}\text { In the source } \\
\text { solution }\end{array}$} & \multicolumn{2}{|c|}{ On the solid-phase matrix } & & \\
\hline & & FIIm & Fiber & Film & Fiber \\
\hline Ethanol $+\mathrm{H}_{2} \mathrm{O}$ & 1.10 & 1.50 & 1.45 & $15 \pm 3$ & $10 \pm 3$ \\
\hline $\mathrm{SDS}+\mathrm{H}_{2} \mathrm{O}$ & 1.12 & 1.04 & 1.09 & $58 \pm 3$ & $39 \pm 3$ \\
\hline $\mathrm{CTAB}+\mathrm{H}_{2} \mathrm{O}$ & 1.15 & 1.02 & 1.06 & $56 \pm 4$ & $33 \pm 4$ \\
\hline $\mathrm{TX}-100+\mathrm{H}_{2} \mathrm{O}$ & 1.25 & 1.09 & 1.10 & $84 \pm 3$ & $83 \pm 3$ \\
\hline
\end{tabular}


CTAB and TX-100 (Fig. 2, curves 1). After sorption from water-micellar media, the fluorescence intensity of the hydrocarbon in all solutions of the surfactants used significantly reduced (Fig. 2, curves 2 and 3).

These results show that pyrene sorption from aqueous micellar solutions of the anionic SDS on the CDA film is more effective in comparison with sorption on the CDA: PEO fibers from the same solution (Fig. 2 a). Similar results were obtained for the cationic CTAB as well (Fig. 2 b). The signal
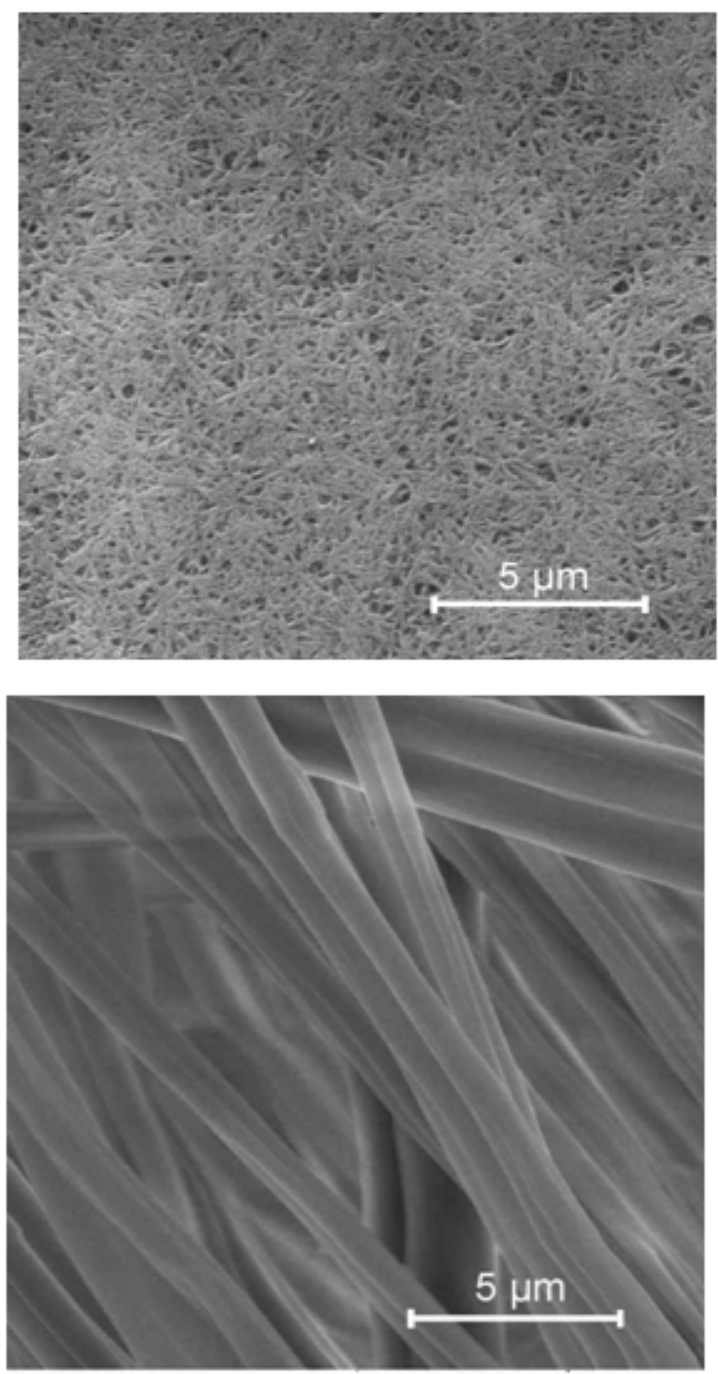

Fig. 1: SEM images of the surface structure of our CDA film (a) and that of our electrospun fibers (CDA : PEO = $90: 10)(b)$
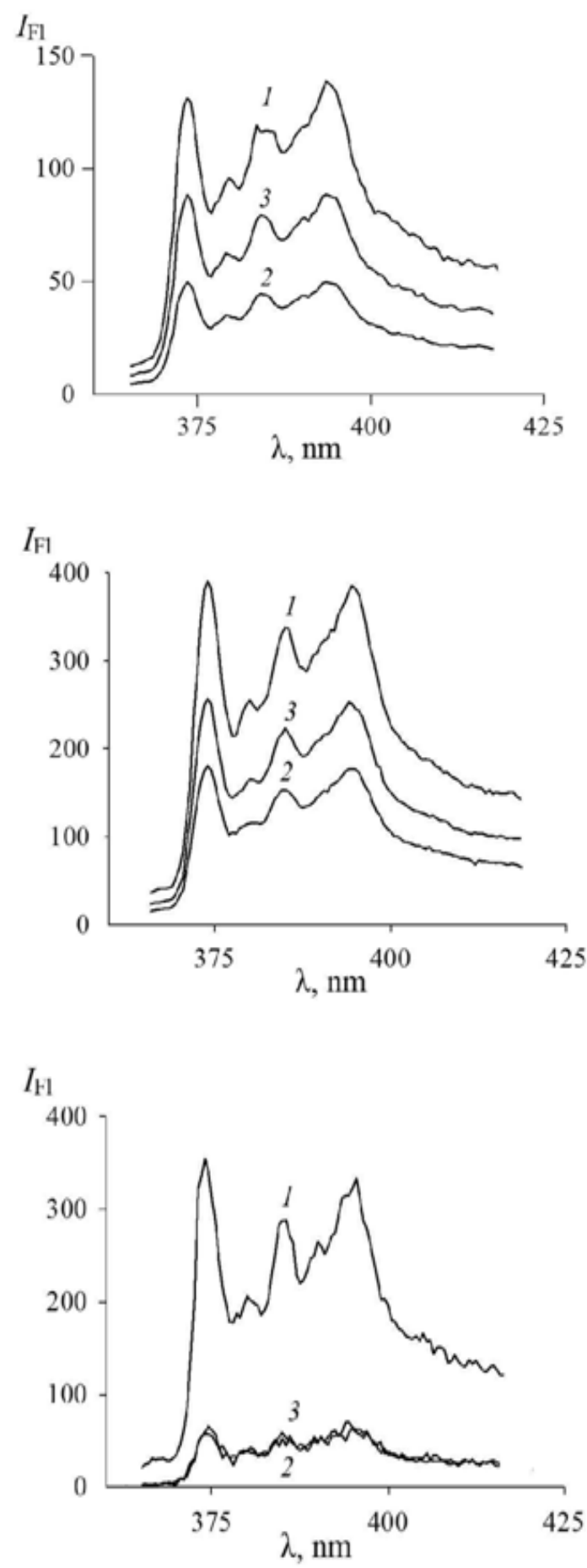

Fig. 2: Fluorescence spectra of pyrene in aqueous micellar solutions of SDS (a), CTAB (b), and TX-100 (c) before (1) and after sorption on the CDA film (2) and on the CDA : PEO = $90: 10$ fibers (3). 
intensity of pyrene in aqueous micellar solutions of CTAB after sorption on the CDA: PEO fibers was higher than that after sorption on the CDA film. Comparison of the fluorescence spectra of pyrene in aqueous micellar solutions of the nonionic TX-100 has revealed that the signal intensity of the probe in the solutions after sorption on both fiber and film has approximately the same value (Fig. 2 c).
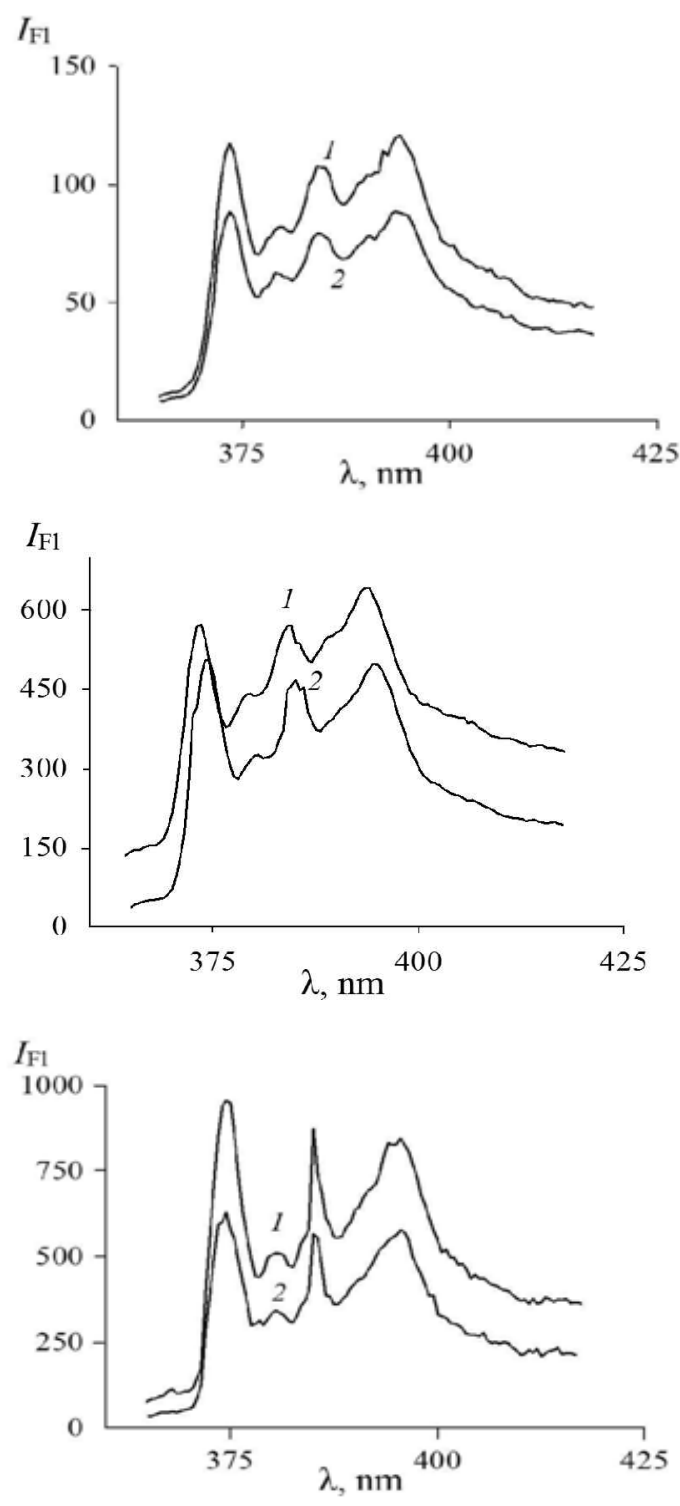

Fig. 3: Fluorescence spectra of pyrene on the CDA film (1) and on the CDA : PEO = $90: 10$ fibers (2), after sorption of this hydrocarbon from water-micellar solutions of SDS (a), CTAB (b), and TX-100 (c)
The fluorescence spectra of pyrene in the sorbent phase modified with anionic, cationic and nonionic surfactants were analyzed.

It was found that the fluorescence intensity of pyrene on the CDA film modified with SDS, CTAB or TX-100 was much higher than the intensity of pyrene fluorescence on the CDA: PEO fibers (Fig. 3). These experiments show once again that when CDA surfactant-modified matrices are used, pyrene interacts better with the film than with the fiber. Probably, this may be due to the different influence of our matrices on the radiative and non-radiative transitions of pyrene on these sorbents.

We hypothesized that these results could be explained by the Coulomb interaction of the particles (surfactant micelles with the solubilized hydrocarbon) due to the charged matrix. For this, the surface potential of the sorbents was estimated. The outer surface of the CDA film has not a large (by magnitude) negative surface potential equal to $-32 \pm 2 \mathrm{mV}$. The CDA: PEO fiber is characterized by a positive value of its surface potential, $+419 \pm 1$ $\mathrm{mV}$, more by an order of magnitude exceeding the absolute value of the film sample's surface potential. This suggests that the positively charged CTAB, forming micelles with the solubilized pyrene, better interact with the negatively charged surface of the film than with the fiber having a positive surface charge.

The similar character of the interaction of pyrene with the CDA film and CDA: PEO fibers in the presence of the negatively charged SDS is probably due to recharging of the polymer matrix surface due to the adsorption of the surfactant anion. For example, a sign change of the electric potential from negative to positive with transition through an isoelectric state was observed in Ref. (19) for cellulose fibers (both unmodified and CDAfilm-coated) in solutions of low- and high-molecularweight surfactants. The inversion of the surface charge on anion adsorption is possible for both film and fibers. This hypothesis is currently being tested.

The relatively high values of the fluorescence intensity of pyrene in the adsorbed state on the CDA film or CDA: PEO fiber should be also noted. It was found that when aqueous 
micellar solutions of CTAB and TX-100 were used as the sorbate the fluorescence intensity of pyrene on the solid-phase matrices was significantly higher (Fig. $3 b, c)$ than in the source solution which the sorption was carried out from (Fig. $2 b, c$, curves 1). This fluorescence intensity increase is evidently associated with a decrease in the probability of non-radiative deactivation of the pyrene molecules' energy due to loss of the mobility of the adsorbed hydrocarbon molecules.

The process of PAH sorption from solutions onto matrices was monitored not only by the fluorescence intensity of the pyrene sorbed onto the film or fibers but also by the polarity index. This is the intensity ratio of the first and third bands in the emission spectrum $\left(I_{1} / I_{3}\right)^{7}$. It was found that during the transition from water-ethanol solutions of pyrene to its aqueous micellar solutions the polarity index of the hydrocarbon in the adsorbed state on the solidphase matrices decreased (Table).

The pyrene extraction degree $(R)$ increased. This indicates that the pyrene sorption from surfactant solutions is more effective as compared to the sorption from ethanolic solutions. The highest sorption activity of our matrices was noted on their TX-100 modification.

\section{CONCLUSION}

From our results we conclude that the modification of our CDA films and fibers with surfactants can significantly increase the sorptive activity of the matrices, increase the sensitivity of the solid-state PAH luminescence technique, simplify the preparation stage of test samples, and reduce the detection limits. Thus, the usage of CDA-based matrices for PAH sorption from aqueous micellar solutions is very promising. The proposed polymer matrices can be used in sensor systems for monitoring aquatic environments, in pharmacological and toxicological studies.

\section{ACKNOWLEDGMENTS}

The results of this work were obtained in the framework of implementation of the state task No 4.1299.2014/K of the Russian Ministry of Education and Science.

The authors thank the staff of the Electrospinning Department of the ERI of nanostructures and biosystems, SSU (Russian Federation, Saratov) for making a sample of nonwoven material from electrospun CDA fibers.

\section{REFERENCES}

1. Goryacheva, I.Y.; Shtykov, S.N.; Loginov, A.S.; Panteleeva, I.V. Anal. Bioanal. Chem. 2005, 382, 6, 1413-1418

2. Wang, H.; Yu, S.; Campiglia, A.D. Anal. Biochem. 2009, 385, 249-256

3. Occello, V.N.S.; Veglia, A.V. Analytica Chimica Acta. 2011, 689, 1, 97-102

4. Wilson, W.B.; Costa, A.A.; Wang, H.; Campiglia, A.D.; Dias, J.A.; Dias, S.C.L. Microchem. J. 2013, 110, 246-255

5. Saitoh, T.; Itoh, H.; Hiraide, M. Talanta. 2009, 79, 177-182

6. Dmitrienko, S.G.; Gurariy, E.Y.; Nosov, R.E.; Zolotov, Y.A. Anal. Lett. 2001, 34, 3, 425-438

7. Dyachuk, O.A.; Gubina, T.I.; Mel'nikov, G.V. J. Anal. Chem. 2009, 64, 1, 7-11

8. Vasquez, V.; Baez, M.E.; Bravo, M.; Fuentes,
E. Anal. Bioanal. Chem. 2013, 405, 7497-7507

9. Romanovskaya, G.I.; Olenin, A.Y.; Vasil'eva, S.Y. Russian J. Phys. Chem. A. 2011, 85, 2, 274-278

10. Wang, H.; Campiglia, A.D. Talanta. 2010, 83, 233-240

11. Dyachuk, O.A.; Gubina, T.I.; Khatuntseva, L.N. Proceedings of the universities. Chemistry and chemical technology. 2006, 49(2), 45-48

12. Pramauro E. and Pelizzetti E., Surfactants in analytical chemistry. applications of organized amphiphilic media, Elsevier, (1996)

13. Zakaria R. Hydrogen separation using asymmetric cellulose acetate hollow fiber membranes, Canada, University of Waterloo, (2006)

14. Shipovskaya, A.B.; Hegel, N. O.; Schogolev, 
S. Yu.; Timofeeva, G.N. Proceedings of the universities. Chemistry and chemical technology. 2007, 50, 3, 19-23

15. He, Y.; Li, G.-M.; Wang, H.; Jiang, Z.-W.; Zhao, J.-F.; Su, H.-X.; Huang, Q.-Y. J. Taiwan Inst. Chem. Eng. 2009, 40, 3, 289-295

16. Zhang, S.; Wang, K.Y.; Chung, T. S.; Chen, H.; Jean, Y.C.; Amy, G. J. Membrane Sci. 2010, $360,1-2,522-535$
17. Baker R.W., Membrane technology and applications, 3 ed.; John Wiley. UK, (2012)

18. Rusli, H.; Gandasasmita, S.; Amran, M.B. Iran. Polym. J. 2013, 22, 5, 335-340

19. Amelina, E.A.; Vidensky, I.V.; Ivanova, N.I.; Parfyonova, A.M.; Pelekh, V.V.; Altukhova, N.V.; Shchukin, E.D. Proc. Moscow. Univ. Ser. 2 Chemistry. 2001, 42, 1, 49-54 